\title{
ZNF667 Serves as a Putative Oncogene in Human Hepatocellular Carcinoma
}

\author{
Ke Chenga Zhizhao Chen ${ }^{a, b}$ Lian Liu ${ }^{a}$ Yujun Zhao ${ }^{a}$ Sheng Zhanga Qiang Wanga \\ Zhenghao Deng ${ }^{d}$ Sipin Tan ${ }^{c}$ Qifa Ye $e^{a, b}$ \\ aTransplantation Center of the Third Xiangya Hospital of Central South University, Research \\ Center of National Health Ministry on Transplantation Medicine Engineering and Technology,

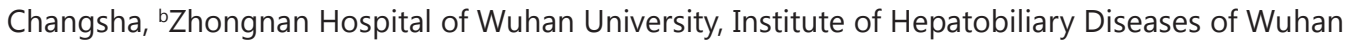 \\ University, Transplant Center of Wuhan University, Hubei Key Laboratory of Medical Technology on \\ Transplantation, Wuhan, 'Laboratory of Shock, Department of Pathophysiology, Xiangya School of \\ Medicine, Central South University, Changsha, 'Department of pathology, school of basic medicine, \\ Central South University, Changsha, China
}

\section{Key Words}

Hepatocellular carcinoma $•$ Zinc finger protein $667 \cdot$ LO2 $\bullet$ HepG2

\begin{abstract}
Background/Aims: Zinc finger protein 667 (ZNF667) is a member of $\mathrm{C} 2 \mathrm{H} 2$ zinc finger protein family. For the first time, we aim to analyze the expression pattern of ZNF667 in hepatocellular carcinoma (HCC) tissues; to explore its role in HCC tumorigenesis. Methods: Immunohistochemistry was carried out to characterize the ZNF667 expression in paraffin-embedded HCC samples. The relationship between ZNF667 expression and the clinical, pathological data of the patients were analyzed. Human normal hepatocyte cells LO2 over expressing ZNF667 (LO2-ZNF667 cells), ZNF667 depleted hepatocellular carcinoma HepG2 cells (HepG2shZNF667 cells) were set up, their proliferation, migration and invasion abilities were analyzed. Xenograft nude mice were used to analyze the malignancy of HepG2-shZNF667 cells in vivo. Western blot was performed to analyze the expression of BCl-2 and BAX in LO2-ZNF667 and HepG2-shZNF667 cells. Results: Increased ZNF667 was found via immuno-histochemistry in HCC. Enhanced ZNF667 expression was associated with tumor size, clinical stage and tumor differentiation. LO2-ZNF667 cells displayed increased and HepG2-shZNF667 cells decreased cell proliferation, migration and invasion. Xenograft experiments proved reduced malignancy of HepG2-shZNF667 cells in vivo. LO2-ZNF667 cells displayed increased Bcl-2 and decreased BAX protein expression. HepG2-shZNF667 cells displayed enhanced BAX and inhibited BCL-2 expression. Conclusions: ZNF667 is shown to be a new oncogene in HCC and it may serve as a new therapeutic target for $\mathrm{HCC}$ via enhancing $\mathrm{BCL}-2$ and decreasing $\mathrm{BAX}$ expression.
\end{abstract}

Q. Ye and S. Tan contributed equally to the work. 


\section{Introduction}

As one of the most common types of liver cancer, the mortality of hepatocellular carcinoma (HCC) has significantly increased over the past decades worldwide [1, 2]. Early diagnosis and surgical resection of HCC increase its survival rate. The overall 5-year survival rate of HCC patients' remains low, however. Thus, identification of new diagnostic mark proves to be important for the early diagnosis of HCC $[3,4]$.

Zinc finger proteins are a superfamily of transcription factors which are expressed in abundance in eukaryotic cells [5]. Quite a few amount of the zinc finger proteins were involved in development of tumors [6]. ZNF165 mRNA and protein were remarkably increased in urinary bladder cancer [7]. ZNF217 enhanced its expression in colorectal adenoma, gastric cancer and ovarian cancer [8,9]. As a member of Kruppel-like transcription factors (KLF), ZNF139 enhanced its expression in gastric cancer via apoptosis dependent pathway [10].

Being a member of C2H2 zinc finger protein family, Zinc finger protein 667 (ZNF667) was initially found to be a significantly over-expressed protein during myocardial ischemic preconditioning $[11,12]$. Enhancing expression of ZNF667 can inhibit the apoptosis via inhibiting Bax and Fas expression $[13,14]$. Much in the same way, as a widely expressed protein in many tissues, increased ZNF667 protein was observed in astrycytoma [15], while the expression of ZNF667 in other types of tumors was not explored. For the first time, immunohistochemistry was performed to detect the expression of ZNF667 in HCC tissues and its relation with the clinical parameters was analyzed. Via transfection of ZNF667-pEGFP plasmid, ZNF667 over expression L02 cell lines were set up, ZNF667 shRNA plasmids were also constructed and tranfected into HepG2 cells. The proliferation, invasion and migration characters of the ZNF667 over expression L02 cells and ZNF667 depleted HepG2 cells were further clarified respectively so as to characterize the role of ZNF667 in HCC.

\section{Materials and Methods}

\section{Human tissue specimens and ethic statement}

A total of 66 paraffin-embedded HCC sample was collected in the Third Xiangya Hospital from January 2014 to May 2015, the sample was histologically and clinically diagnosed in patients with radical surgery. None of the patients had received radiotherapy or chemotherapy prior to surgery. Clinical and pathological data of the 66 patients with HCC, including age, tumor size, stage, differentiation grade, Hepatitis B infection and Cirrhosis, was collected. The tumor stages were classified according to the 2010 TNM staging system of Union for International Cancer Control (UICC). Tumor differentiation was classified according to the Edmondson grading system. Both written Ethics Approval and Patient Consent were obtained from the Research Ethics Committee of the Third Xiangya Hospital and Central South University. Written informed consent was also obtained from all the participants involved in the study.

\section{Immunohistochemical analyses}

Specimens were fixed with $10 \%$ formaldehyde and embedded in paraffin blocks. Sections (4 $\mu \mathrm{m}$ ) were deparaffinized with xylene, rinsed and rehydrated through a graded series of alcohol. Immunohistochemistry was performed as described previously [16]. Staining reactions were determined by microscopic examination. Both the intensity and extent of staining were taken into account when data analyzed. The extent of staining was scored from 0 to $100 \%$ (1 indicates <10\%, 2 indicates $10-50 \%, 3$ indicates $>50 \%$ ), and the intensity of staining was scored from 0 to 3 ( 0 indicates none; 1 indicates weak; 2 indicates moderate; 3 indicates strong). IHC score= extent of staining $\times$ intensity of staining. The expression level was determined as follows: high expression: IHC score $\geq 6$; low expression: IHC score $\leq 5$.

Cell lines and Plasmids

Human hepatocyte (LO2) and hepatocellular carcinoma cells (HepG2) were purchased from Cell Biology Laboratory of Central South University. LO2 and HepG2 cells were cultured in high-glucose Dulbecco's modified Eagle's medium (Gibco, Grand Island, NY, USA) supplemented with 10\% fetal bovine 


\section{Cellular Physiology Cell Physiol Biochem 2017;41:2523-2533 \begin{tabular}{l|l|l} 
and Biochemistry $10.1159 / 000475971$ & $\begin{array}{l}\text { DO } 2017 \text { The Author(s). Published by S. Karger AG, Basel } \\
\text { Published onme:karger.com/cpb }\end{array}$
\end{tabular} \\ Cheng et al.: Exploration of ZNF667 in HCC}

Serum (FBS, Invitrogen, CA, USA) and $1 \%$ penicillin-streptomycin mixture (Invitrogen, CA, USA) at $37^{\circ} \mathrm{C}$ and were maintained in a humidified environment containing $5 \% \mathrm{CO}_{2}$.

The plasmids pEGFP-ZNF667 and ZNF667 shRNA plasmids were constructed as described previously [17]. In brief, the ORF of ZNF667 was subcloned into pEGFP plasmid. The siRNA sequence (CGCCAATCATTTCTTATTGAA), which matches 1549-1570 nucleotide of ZNF667 (NM_022103.3), was subcloned into eukaryotic expression vectors purchased from Genechem (Shanghai, China, containing hU6MCS-CMV-GFP-SV40-Neomycin elements) to construct shRNA.

Western blotting

Protein was extracted from the indicated cells using RIPA lysis buffer. Protein assay was performed as described previously. Equal amount of protein lysates was separated by SDS-PAGE and transferred onto nitrocellulose membranes. Filters were probed with the following specific primary antibodies: rabbit antihuman ZNF667 polyclonal antibody (ab106432), anti-human Bcl-2 polyclonal antibody (ab117115) and anti-human BAX polyclonal antibody (ab32503) purchased from Abcam (Cambridge, England). Blots were then incubated with horseradish peroxidase-conjugated secondary antibody (Boster Biotechnologies) and visualized by chemiluminescence. The band density was quantified by densitometry using Scion Image software, and normalized to $\beta$-actin levels.

\section{Cell proliferation assay}

Cell viability was detected by Cell Counting Kit-8 (CCK-8, Beyond time, China). The assay was performed as described previously and repeated three times.

\section{Plate colony formation assay}

Plate colony formation assay was performed as described previously, Clone formation efficiency was calculated according to the formula: (the clone number/the plated cell number) $\times 100 \%$.

\section{In vitro wound healing assay}

For wound healing assay, the L02, LO2-ZNF667 and LO2-NC cells; the HepG2, HepG2-shZNF667 and HepG2-NC were seeded in 24 -well plates $\left(1 \times 10^{5}\right.$ cells/well). After reaching appropriate $90 \%$ confluency, a $200 \mu \mathrm{l}$ tip was performed to produce a wound line on the cell monolayer. Images were captured at different time points by microscopy to assess the rate of gap closure. The distance between two edges was measured by Image J software. At the bottom of each dish, three arbitrary places were marked where the width of the wound was measured with an inverted microscope (objective $\times 10$ ). Representative images of cells migrated into the wounds were captured and the length of the scratch was recorded and calculated $48 \mathrm{hr}$ later. The results show that wound closure rate $=(0 \mathrm{hr}$ scratch width $-48 \mathrm{hr}$ scratch width $/ 0 \mathrm{hr}$ scratch width $\times 100$ [18].

\section{Transwell invasion assay}

The invasion ability of the three HepG2 and three LO2 cells was detected by Transwell assay separately. Cells were plated in 24-well plates $\left(1 \times 10^{5}\right.$ cells/well, bottom chamber, $8 \mu \mathrm{m}$ pore size with Matrigel-coated membranes). Serum-free medium was added into the bottom chamber. The bottom chamber contained 700 $\mu \mathrm{l}$ medium with $20 \%$ FBS. After $24 \mathrm{~h}$, the total numbers of the Transwell assay were imaged and the number of invasive cells was evaluated by measurement of absorbance measurement at $570 \mathrm{~nm}$ [18].

\section{Subcutaneous tumor model}

The animal experiments were approved by the Ethics Committee of The Third Xiangya Hospital, Central South University. Four to five week old BALB/c nude mice (Shanghai SLAC Laboratory Animal Co. Ltd., Shanghai, China) were used in the nude mouse tumorigenesis assay. Nude mice were randomly separated into the parental cell group, control vector group or the shZNF667 group. Cells $\left(2 \times 10^{6}\right.$ cells $/$ mouse) stably transfected with vectors were injected subcutaneously into the right axilla (200 $\mu \mathrm{l})$. All nude mice were sacrificed at 42 days after tumor implantation, xenografts were dissected and tumor volume and weight were assessed. Tumor volume was calculated according to the following formula: volume $=0.5 \times a \times b^{2}$, where a stands for longest diameter and $\mathrm{b}$ is the transverse diameter, respectively. 


\section{Cellular Physiology Cell Physiol Biochem 2017;41:2523-2533 \begin{tabular}{l|l} 
DOI: 10.1159/000475971 & O 2017 The Author(s). Published by S. Karger AG, Basel \\
www.karger.com/cpb
\end{tabular} \\ Cheng et al.: Exploration of ZNF667 in HCC}

Statistical analyses

All experiments were repeated at least three times. Statistical analyses were made with SPSS 17.0 software (SPSS, Inc., Chicago, IL, USA) and GraphPad Prism 6 (GraphPad Software Inc., CA, USA). Differences between groups were analyzed using the $\chi^{2}$ test, Fishers exact test or one-way analysis of variance. Multivariate survival analysis was done with the Cox multivariate analysis model, according to which $P<0.05$ was considered statistically significant.

\section{Results}

Increased ZNF667 in HCC was associated with clinical stage and cancer differentiation

Immunohistochemistry analyses were made in 66 paraffin-embedded HCC sections and their adjacent normal tissues. Increased ZNF667 expression in HCC was localized in both the nucleus and cytoplasm, with a predominant expression in the nucleus. In our immunohistochemistry work, the most poorly differentiated HCC cases showed the highest ZNF667 expression, moderately-differentiated cases weakly positive expression, while the normal hepatic tissues the weakest ZNF667 expression (Fig. 1A-1D). Statistical analyses showed that the expression of ZNF667 was remarkably up-regulated in HCC tissues

compared with adjacent Table 1. Correlation between the expression of ZNF667 and clinicopanormal tissues (Fig 1E). Based on the categories which we defined in the previously mentioned methods, the data showed that higher expression of ZNF667 was significantly associated with tumor size $\quad(P=0.006)$, clinical stage $(P=0.046)$ and tumor differentiation $(P=0.047)$, whereas no significant relevance was found with age, sex distribution, hepatitis $B$ infection and cirrhosis (shown in Table 1). The analysis results suggested that ZNF667 may play a vital role in the cirrhosis development of HCC.

\begin{tabular}{|c|c|c|c|c|}
\hline \multirow{2}{*}{ Factors } & & \multicolumn{2}{|c|}{ ZNF667 expression } & \multirow{2}{*}{$P$ value } \\
\hline & & Higer expression $(n=33)$ & Lower expression $(n=33)$ & \\
\hline \multirow[t]{3}{*}{ Age (yr) } & & & & 0.804 \\
\hline & $\leq 50(\mathrm{n}=29)$ & 14 & 15 & \\
\hline & $>50(n=37)$ & 19 & 18 & \\
\hline \multirow[t]{3}{*}{ Sex distribution } & & & & 0.802 \\
\hline & Female $(n=27)$ & 14 & 13 & \\
\hline & Male $(n=39)$ & 19 & 20 & \\
\hline \multirow[t]{3}{*}{ Tumor size } & & & & $0.006^{*}$ \\
\hline & $\leq 5 \mathrm{~cm}(\mathrm{n}=29)$ & 9 & 20 & \\
\hline & $>5 \mathrm{~cm}(\mathrm{n}=37)$ & 24 & 13 & \\
\hline \multirow[t]{3}{*}{ clinical stages } & & & & $0.046^{*}$ \\
\hline & I-II $(\mathrm{n}=38)$ & 15 & 23 & \\
\hline & III-IV $(n=28)$ & 18 & 10 & \\
\hline \multirow[t]{4}{*}{ Differentiation } & & & & $0.047^{*}$ \\
\hline & Well $(n=16)$ & 5 & 11 & \\
\hline & Moderate $(n=27)$ & 12 & 15 & \\
\hline & Poor $(n=23)$ & 16 & 7 & \\
\hline \multirow[t]{3}{*}{ Hepatitis B infection } & & & & 0.251 \\
\hline & Positive $(n=50)$ & 27 & 23 & \\
\hline & Negative $(n=16)$ & 6 & 10 & \\
\hline \multirow[t]{3}{*}{ Cirrhosis } & & & & 0.614 \\
\hline & Positive $(\mathrm{n}=26)$ & 14 & 12 & \\
\hline & Negative $(n=40)$ & 19 & 21 & \\
\hline
\end{tabular}

Fig. 1. Increased ZNF667 expression in HCC tissues via immunohistochemistry. A. Normal hepatic tissues, scored as ZNF667 (-); B. Well-differentiated hepatic cancer, scored as ZNF667 (+); C. Moderately differentiated hepatic cancer, scored as ZNF667 (++); D. Poorly differentiated hepatic cancer, scored as ZNF667 (+++). Original ma-
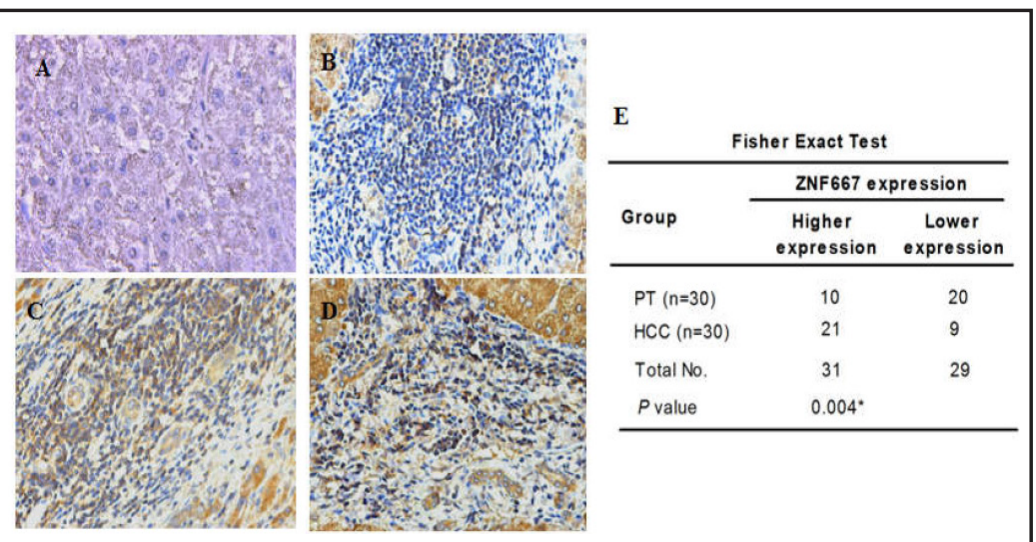
gnifcation: A-D ×400. E. Statistical analyses between HCC tissues and para-carcinoma tissues (PT).

\section{KARGER}


Fig. 2. Increased ZNF667 in LO2 cells alters its biological traits. A. Western blot performed displayed enhanced ZNF667 expression in L02ZNF667 cell lines. Statistical analyses proved that the difference were significant. B. CCK-8 assays of three LO2 cell lines, showing that LO2ZNF667 cell proliferate faster than LO2-NC and blank LO2 cells at the indicated time points, the difference was statistically significant, ${ }^{*} P<0.05$. C. Clone formation assays of three LO2 cell lines. The colony forming efficiency of LO2ZNF667 cell was larger than LO2-NC and blank LO2 cells at the indicated time points, the difference was statistically significant, ${ }^{*} P<0.05$. D. Wound closure assay of three LO2 cell lines. The width of the scratch at $48 \mathrm{~h}$ was shorter for L02-ZNF667 cell, compared with LO2-NC and blank LO2 cells. The difference was

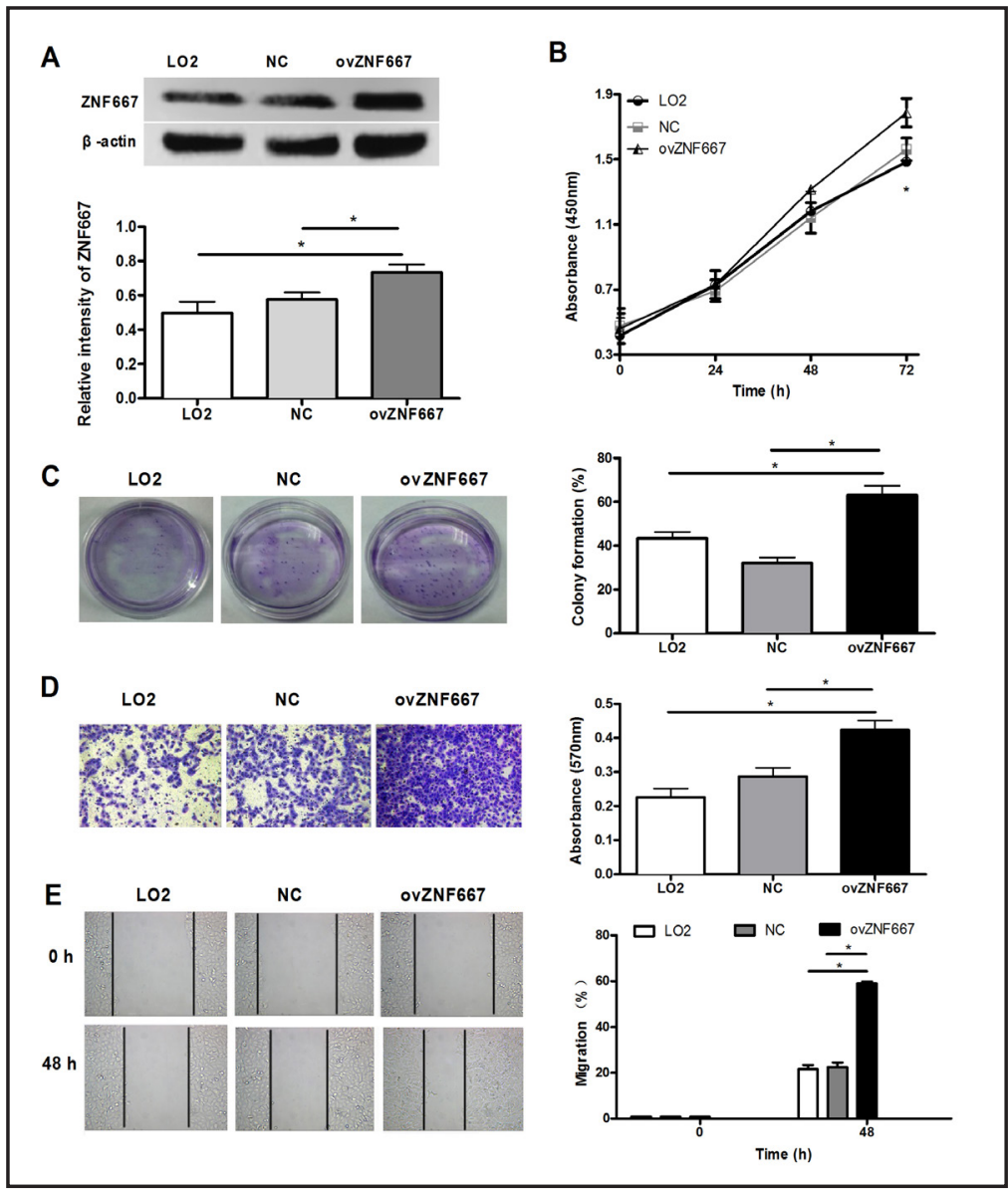
statistically significant, ${ }^{*} P<0.05$. E. Transwell analyses of three LO2 cell lines. Representative micrographs of the transwell invasion assay (10× magnifications). The statistical analysis revealed that the differences between the LO2-ZNF667 cells, LO2 and LO2-NC cells are significant.

\section{LO2-ZNF667 cells displayed increased proliferation and clone formation efficiency}

LO2 cells stably transfected with ZNF667 were used to investigate biological traits of increased ZNF667 in HCC. Increased ZNF667 expression in L02-ZNF667 cells detected by western blot proved the success of stable ZNF667 transfection (Fig. 2A). CCK-8 assay was performed to detect the proliferation capability of L02-ZNF667 cells, as indicated by Fig2B, the L02-ZNF667 cells proliferate faster than LO2 transfected with empty vectors and parental cells $(P<0.05)$. Colony formation assay performed also proved enhanced colony formation of L02-ZNF667 cells (Fig. 2C). CCk8 and colony formation assay proved that increased ZNF667 can significantly enhance the proliferation of LO2 cells.

\section{L02-ZNF667 cells displayed increased invasion and migration capabilities}

Transwell and wound closure assays were performed to measure the migration capabilities of the L02-ZNF667 cells. As indicated in Fig. 2D and 2E, the L02-ZNF667 cells displayed statistically enhanced migration and invasion capabilities. The results displayed that enhancing ZNF667 in L02 can significantly alter their proliferation, migration and invasion capabilities.

ZNF667 depleted HepG2 cells displayed inhibited proliferation and clone formation

ZNF667-shRNA was transfected into HepG2 cells to further clarify its oncogene traits. As indicated in Fig 3A, Western blot proved the success of stable ZNF667 -shRNA transfection. CCK8 displayed that HepG2-ZNF667shRNA grew slower than the parental and HepG2-NC 
A

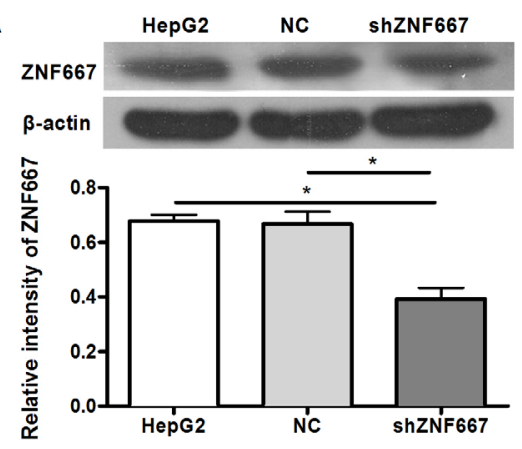

C

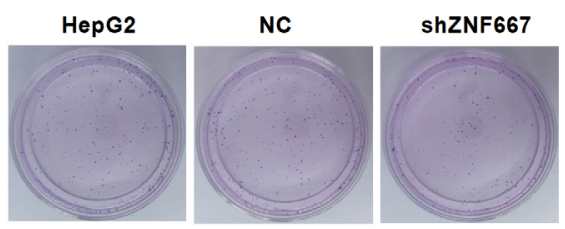

D

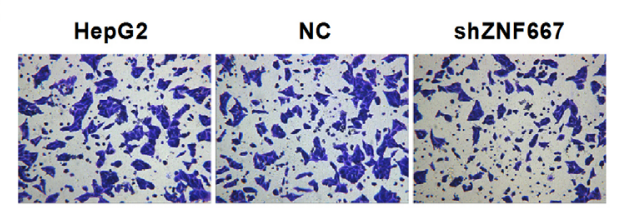

$\mathbf{E}$
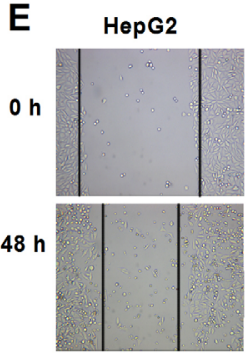

NC

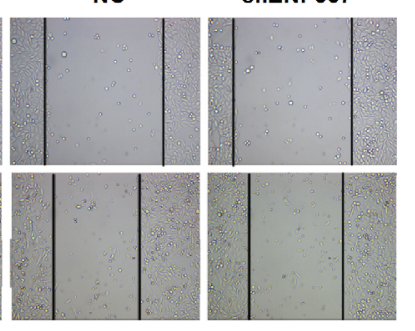

B
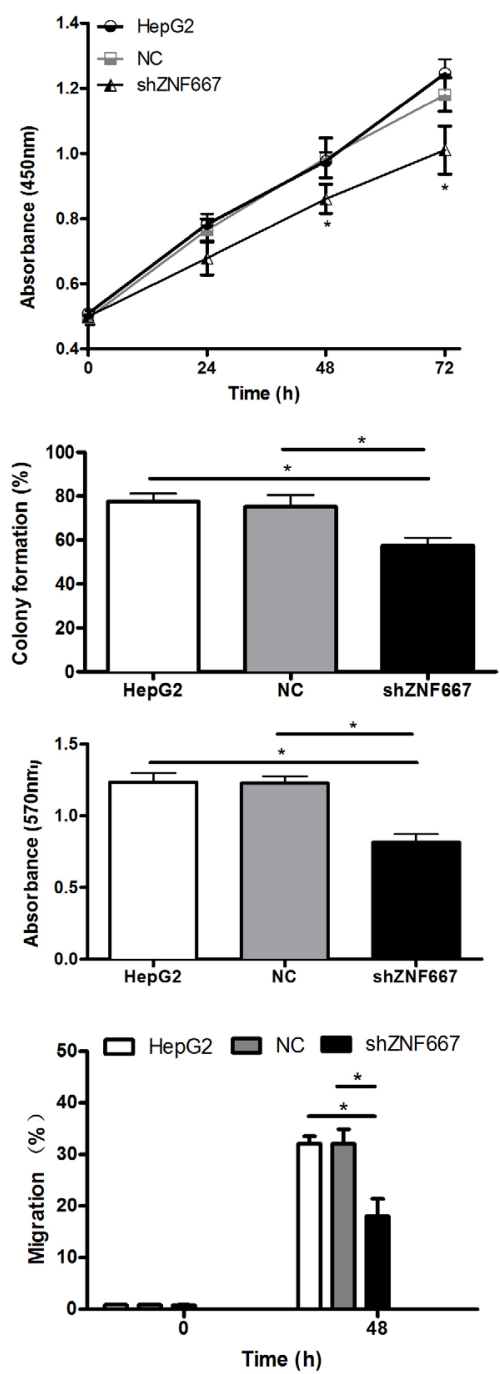

Fig. 3. Knocking down ZNF667 in HepG2 reduces its malignancy in vitro. A. Western blot performed displayed decreased ZNF667 expression in HepG2-shZNF667 cells. Statistical analyses proved that the difference was significant. B. CCK-8 assays of three HepG2 cell lines, showing that HepG2-shZNF667 cell proliferate slower than HepG2-NC and blank HepG2 cells at the indicated time points, the difference was statistically significant, $* P<0.05$. C. Clone formation assays of three HepG2 cell lines. The colony forming efficiency of HepG2-shZNF667 cell was smaller than HepG2-NC and blank HepG2 cells at the indicated time points, the difference was statistically significant, ${ }^{*} P<0.05$. D. Wound closure assay of three HepG2 cells. The width of the scratch at 48h was greater for HepG2-shZNF667 cell, compared with HepG2-NC and blank HepG2 cells. The difference was statistically significant, ${ }^{*} P<0.05$. E. Transwell analysis of three HepG2 cell lines. Representative micrographs of the transwell invasion assay (10× magnifications). The statistical analyses revealed that the differences between the HepG2-shZNF667 cells, HepG2 and HepG2-NC cells are significant.

cells (Fig. 3B). Clone formation assay in Fig 3C also proved that the depletion of ZNF667 can inhibit the growth capability of HepG2 cells.

HepG2- shZNF667 cells displayed inhibited migration and invasion capabilities

Transwell and wound closure assays were performed to measure the invasion and migration capabilities of HepG2-shZNF667. Figure 3D and 3E showed that knocking down 


\section{Cellular Physiology Cell Physiol Biochem 2017;41:2523-2533 \begin{tabular}{l|l|l} 
DOI: 10.1159/000475971 & O 2017 The Author(s). Published by S. Karger AG, Basel \\
www.karger.com/cpb
\end{tabular}

Fig. 4. Knocking down ZNF667 in HepG2 reduces its malignancy in vivo. A. Growth curve of nude mice with three different HepG2 cells. B. Representative images of subcutaneous tumors at 42nd day. Tumor volume (C) and mass (D) of HepG2 cell xenografts in the different groups. D. Western blot was performed to confirm the expression level of ZNF667 in tissues of xenograft tumors. The differences were statistically significant, ${ }^{*} P<0.05$.

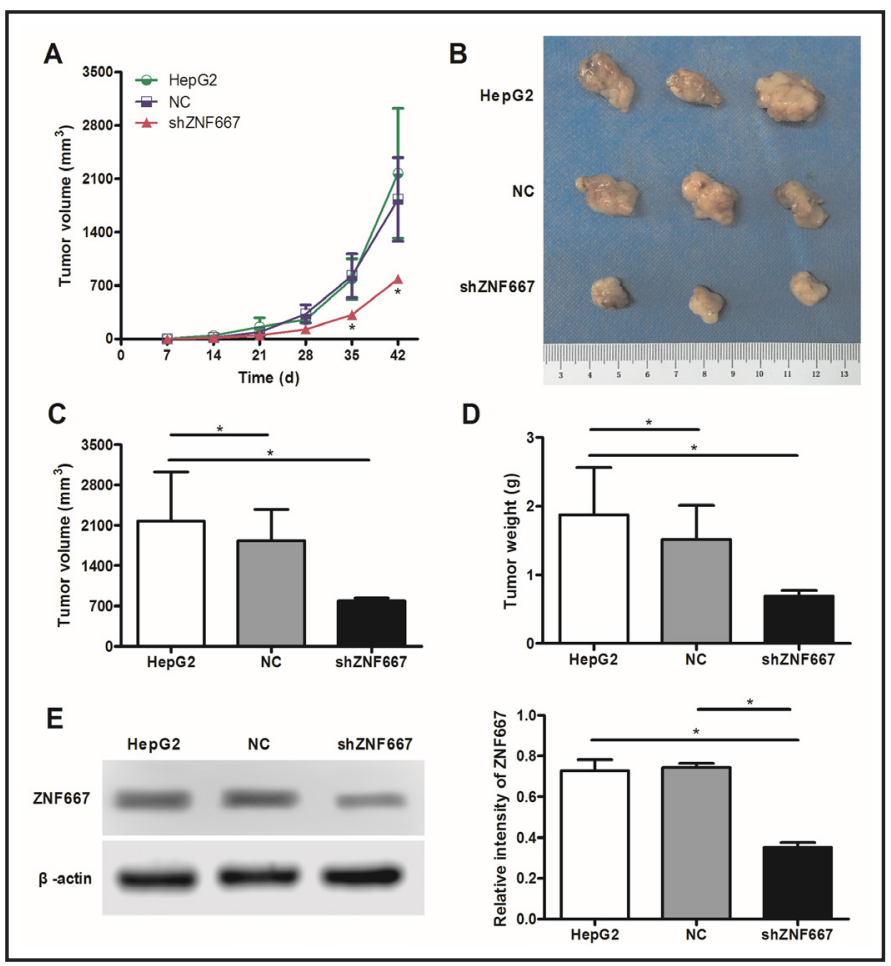

ZNF667 in HepG2 cells inhibit their invasion and migration capabilities significantly. Taking together the proliferation and clone formation assays, we conclude that knocking ZNF667 down in HepG2 cells can successfully reduce their malignancy in vitro.

ZNF667 depleted HepG2 cells displayed reduced malignancy in vivo

In vivo xenograft experiments were performed to validate the effect of the decreased ZNF667 expression in HepG2 cells in vivo. HepG2 cells stably transfected with the ZNF667shRNA vector or blank empty (NC) vector were subcutaneously inoculated into the right axilla of nude mice ( $n=8$ per group). Knocking down ZNF667 in HepG2 significantly inhibit their growth in vivo, the growth curve of each group showed at rapid increase in the tumor volumes at the 28th day in the untreated and NC groups, compared with the ZNF667 knocking down HepG2 cells (Fig. 4A). By the end of the experiment, the mean size $\left(788.533 \pm 53.860 \mathrm{~mm}^{3}\right)$ and weight $(0.693 \pm 0.078 \mathrm{~g})$ of ZNF667 knockout tumors were significantly smaller than those of HepG2 $\left(2174.459 \pm 851.893 \mathrm{~mm}^{3}, 1.873 \pm 0.692 \mathrm{~g}\right)$ and empty vector-transformed tumors $\left(1829.939 \pm 549.144 \mathrm{~mm}^{3}, 1.517 \pm 0.497 \mathrm{~g}\right)$ respectively (Fig. 4C\&4D). The differences above indicate that reduction of ZNF667 protein can successfully suppress tumor growth in vivo.

\section{LO2-ZNF667 cells displayed increased Bcl-2 and decreased BAX protein expression}

Previous research proved transcriptional regulation of BAX by ZNF667 in H9C2 cells. Bax is considered as a good indicator of the apoptotic activity of tumor cells. The crosstalk between bcl-2 and BAX exists in many different tumors $[19,20]$. Western blotting was performed to analyze the expression of Bcl2 and Bax in L02-ZNF667 cells, NC cells and parental cells. As indicated in Figure 5A, compared with $\mathrm{NC}$ cells and parental cells, significantly increased Bcl2 expression and decreased BAX were detected in LO2-ZNF667 cells.

ZNF667 depleted HepG2 cells displayed enhanced BAX and inhibited BCL-2 expression

Western blot was performed to detect the Bcl-2 and Bax expression in the three HepG2 cells. The results in Fig. 5B showed that Bcl-2 expression was inhibited while BAX expression was increased in HepG2-shZNF667 cells (Fig. 5B, $P<0.05$ ). The WB results in the three LO2 


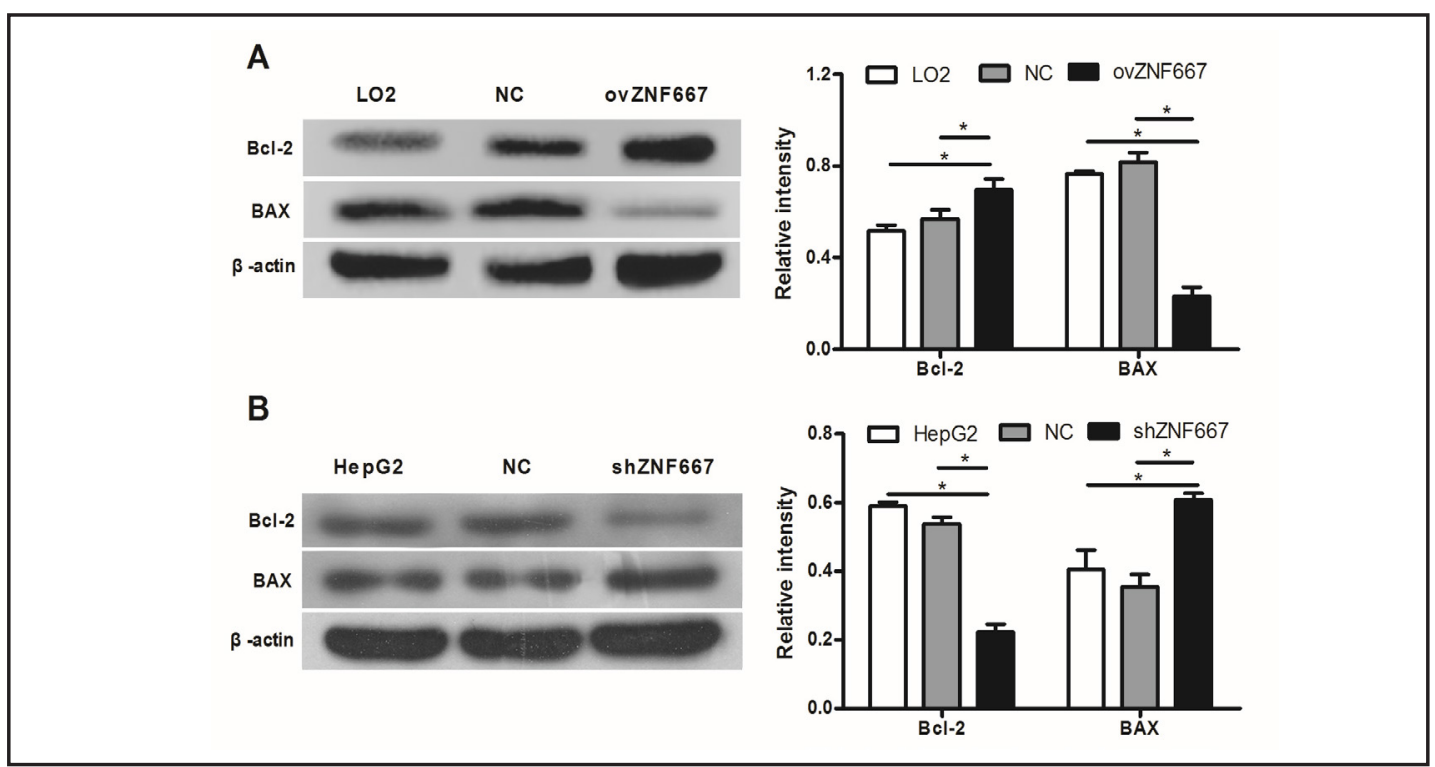

Fig. 5. Alteration of BCL-2 and Bax expression in L02-ZNF667 cells and HepG2-shZNF667 cells. A. In L02ZNF667 cells, increased Bcl-2 and decreased Bax expression was displayed, the expression difference between the three LO2 cell lines was statistically significant, ${ }^{*} P<0.05$. B. Decreased Bcl-2 and increased Bax expression was detected in HepG2-shZNF667 cells by western blot, the expression differences between the three HepG2 cell lines were statistically significant, ${ }^{*} P<0.05$.

and HepG2 cells displayed that Bcl-2 and BAX are two putative downstream molecular targets for the putative oncogenic function of ZNF667.

\section{Discussion}

Zinc finger proteins were proved to play an important role in tumorigenesis. ZFP91-P increased its expression in pancreatic cancer. Via increasing the expression of $\beta$-catenin and decreasing the expression of vimentin, knocking down ZFP91-P significantly inhibited cell proliferation and migration [21, 22]. ZNF309 was firstly found up-regulated its expression in aggressive colorectal cancer. The researchers found that down-regulation of ZKSCAN3 significantly inhibited tumor growth in colon cancer cells, whereas overexpression of ZKSCAN3 produced the opposite effect. In addition, ZKSCAN3 was found to be highly expressed in multiple myeloma [23, 24] and prostate cancer [25]. Through transcriptional activation of cyclin D2 expression, ZKSCAN3 enhanced tumor cell proliferation. It is necessary to notice that a recent study found that ZKSCAN3 also play a role of autophagy in many kinds of tumors, including cervical cancer, colon cancer, neuroblastoma, and ovarian cancer cell [26].

Located on chromosome Chr19q13.43 with a KRAB domain in the N-terminus, ZNF667 was found up-regulating its expression in myocardial ischemic preconditioning. Via directly regulating the mRNA and protein expression of Bax, increased ZNF667 in H9C2 cells can resist the apoptosis induced by $\mathrm{H}_{2} \mathrm{O}_{2}$. Increased human ZNF667 was found in brain astrocytomas [12-14], however, the expression of ZNF667 in other different tumor and disease remains to be further explored.

For the first time, increased ZNF667 was observed in HCC via immunohistochemistry. The enhanced ZNF667 was associated with clinical stage and cancer differentiation. L02 cells transfected with ZNF667-pEGFP were set up to further clarify the biological traits of increased ZNF667. Enhanced proliferation, migration and invasion were observed in L02- 
ZNF667 cells. On the contrary, knocking down the ZNF667 in HepG2 cells inhibited their proliferation, migration and invasion capabilities. Xenograft experiments were performed to further clarify the oncogene function of ZNF667 in vivo. The tumor growth curve and final weight, volumes were consistent with the HepG2 cell model. Taken together, these findings suggest that reducing ZNF667 expression significantly inhibited HCC development.

In the further characterization of ZNF667 in other cancer cells, we found that ZNF667 also displayed a high expression pattern in A549 and HeLa, in which the information suggests a highly putative oncogenic role of ZNF667 in lung cancer and cervical cancer as well.

BAX is a proapoptotic member of the bcl-2 family. Loss of Bax expression was detected in breast cancer. Decreased Bax expression was found to associate with shorter breast cancer survival. Increased Bax expression can prolong the patients' survival in ovarian cancer [27, 28]. Cecropin-P17 significantly suppresses hepatic tumor growth in vivo by stimulating $\mathrm{BAX}$ and inhibition Bcl-2 expression [29]. The in vivo and in vitro binding assay proved that ZNF667 can directly bind to the Bax promoter [12]. Western blot of BAX in L02-ZNF667 cells and HepG2-shRNA cells were consistent with previous transcriptional regulation research of ZNF667. Our WB proved that BAX was also a downstream molecule for the pro-tumor function of ZNF667.

Bcl-2 is a molecule which is known for its anti-apoptotic property. Bcl-2 also exerts other important functions including the regulation of the cell cycle, modulation of cell differentiation, and the regulation of gene expression. The coordinate expression of Bcl2 and Bax is crucial for the survival and apoptosis of many different cells. The crosstalk between bcl-2 and BAX exists in hepatic cancer as well [30,31]. Previous research has identified overexpression of Bcl-2 relating to the malignant progression of different tumors. Significant enhanced Bcl-2 expression was detected in LO2-ZNF667 cells. In HepG2-shRNA cells, much less Bcl-2 expression was displayed compared with the parental cells and cells transfected with control plasmids. Our experiments also proved that enhanced ZNF667 can resist the reduced expression of Bcl-2 under $\mathrm{H}_{2} \mathrm{O}_{2}$ (unpublished data) stimulation. The altered expression of Bcl-2 in the LO2 and HepG2 cells highly suggests that it is under transcriptional control of ZNF667. However, bioinformatics analyses failed to identify a putative ZNF667 binding site (the DNA binding sequence is TGTCTTATCGAA, with TCTTA as the core sequence) in -3000-nt region of the ZNF667 5-flanking region. Different from other Zinc finger proteins, ZNF667 is localized in the nucleus by its KRAB domain, therefore, more than eight zinc fingers can be used for protein-protein interactions and/or RNA protein interactions [32-36]. ZNF667 may alter the Bcl2 expression by its interactions with other molecules which may transcriptionally regulate Bcl-2 expression. For the sake of accuracy, however, further experiments are needed to perform to identify the putative binding partners of ZNF667.

The bioinformatics analyses revealed that 53 was also possibly under the transcriptional control of ZNF 667.Two putative ZNF667 binding sites were found in the 5'-UTR of p53( - 421 416, - 539 - 544). Functioning as a tumor suppressor by removing the proliferative or survival capacity of cells with DNA damage or inappropriate cell-cycle progression, p53 has been described as "the guardian of the genome" [37, 38]. The experiments also proved that over expression of ZNF667 could inhibit the increase of p53 in Dox-induced H9c2 cells (unpublished data). Via suppressing the cell cycle arrest, DNA repair function of p53, the enhanced ZNF667 in normal hepatocyte cells can accelerate the liver tumorigenesis. The HCC tumor growth can be treated by chemical compound via interfering different signaling pathways $[39,40]$, the transcriptional control of P53 by ZNF667 suggests its highly putative target for HCC treatment.

To sum up, our study demonstrated that ZNF667 expression is markedly up-regulated in HCC tissues and cell lines. ZNF667 promote the cell proliferation, invasion and migration mainly via interference expression of Bcl-2 and BAX. These findings indicate that ZNF667 serves as a novel prognostic marker and it may serve as a promising molecular target for anti-cancer therapy in HCC and other type of cancer. 


\section{Cellular Physiology Cell Physiol Biochem 2017;41:2523-2533 \begin{tabular}{l|l} 
DOI: 10.1159/000475971 & and Biochemistry \\
Publisned online:1vay U4, 2017 & $\begin{array}{l}\text { O 2017 The Author(s). Published by S. Karger AG, Basel } \\
\text { www.karger.com/cpb }\end{array}$
\end{tabular} \\ Cheng et al.: Exploration of ZNF667 in HCC}

\section{Funding}

This work was supported by Project of Natural Science Foundation of Hunan Province of China (No. 13JJ3036); Technology Project of Hunan Province of China (2012SK3225).

\section{Disclosure Statement}

The authors declare no conflict of interests.

\section{Reference}

1 Chen HA, Kuo TC, Tseng CF, Ma JT, Yang ST, Yen CJ, Yang CY, Sung SY, Su JL: Angiopoietin-like protein 1 antagonizes MET receptor activity to repress sorafenib resistance and cancer stemness in hepatocellular carcinoma. Hepatology 2016;64:1637-1651.

-2 Zheng C, Li J, Wang Q, Liu W, Zhou J, Liu R, Zeng Q, Peng X, Huang C, Cao P, Cao K: MicroRNA-195 functions as a tumor suppressor by inhibiting CBX4 in hepatocellular carcinoma. Oncol Rep 2015;33:1115-1122.

-3 Balogh J, Victor D, 3rd, Asham EH, Burroughs SG, Boktour M, Saharia A, Li X, Ghobrial RM, Monsour HP, Jr: Hepatocellular carcinoma: a review. J Hepatocell Carcinoma 2016;3:41-53.

4 Zhang L, Jia G, Shi B, Ge G, Duan H, Yang Y: PRSS8 is Downregulated and Suppresses Tumour Growth and Metastases in Hepatocellular Carcinoma. Cell Physiol Biochem 2016;40:757-769.

5 Nie HF, Li Y, Li ZX, Mu JX, Wang JS: Effects of ZNF139 on gastric cancer cells and mice with gastric tumors. Oncol Lett 2016;12:2550-2554.

6 Turkel N, Portela M, Poon C, Li J, Brumby AM, Richardson HE: Cooperation of the BTB-Zinc finger protein, Abrupt, with cytoskeletal regulators in Drosophila epithelial tumorigenesis. Biol Open 2015;4:1024-1039.

-7 Singh PK, Srivastava AK, Dalela D, Rath SK, Goel MM, Bhatt ML: Frequent expression of zinc-finger protein ZNF165 in human urinary bladder transitional cell carcinoma. Immunobiology 2015;220:68-73.

-8 Rooney PH, Boonsong A, McFadyen MC, McLeod HL, Cassidy J, Curran S, Murray GI: The candidate oncogene ZNF217 is frequently amplified in colon cancer. J Pathol 2004;204:282-288.

-9 Shida A, Fujioka S, Kurihara H, Ishibashi Y, Mitsumori N, Omura N, Yanaga K: Prognostic significance of ZNF217 expression in gastric carcinoma. Anticancer Res 2014;34:4813-4817.

10 Huang HN, Huang WC, Lin CH, Chiang YC, Huang HY, Kuo KT: Chromosome 20q13.2 ZNF217 locus amplification correlates with decreased E-cadherin expression in ovarian clear cell carcinoma with PI3KAkt pathway alterations. Hum Pathol 2014;45:2318-2325.

11 Yuan D, Huang J, Yuan X, Zhao J, Jiang W: Zinc finger protein 667 expression is upregulated by cerebral ischemic preconditioning and protects cells from oxidative stress. Biomed Rep 2013;1:534-538.

12 Jiang L, Tang D, Wang K, Zhang H, Yuan C, Duan D, Xiao X: Functional analysis of a novel KRAB/C2H2 zinc finger protein Mipu1. Biochem Biophys Res Commun 2007;356:829-835.

13 Jiang L, Wang H, Shi C, Liu K, Liu M, Wang N, Wang K, Zhang H, Wang G, Xiao X: ZNF667/Mipu1 is a novel anti-apoptotic factor that directly regulates the expression of the rat Bax gene in H9c2 cells. PLoS One 2014;9:e111653.

14 Wang G, Jiang L, Song J, Zhou SF, Zhang H, Wang K, Xiao X: Mipu1 protects H9c2 myogenic cells from hydrogen peroxide-induced apoptosis through inhibition of the expression of the death receptor Fas. Int J Mol Sci 2014;15:18206-18220.

15 Wang Z, Gu L, Liu H, Wang D, Tu Z, Wang K, Jiang B, Xiao X: Expression of Mipu1 in human brain astrocytomas and its clinical significance. Chin J Neurosurg Dis Res 12:37-40.

16 Tan S, Tan J, Tan S, Zhao S, Cao X, Chen Z, Weng Q, Zhang H, Wang K, Zhou J, Xiao X: Decreased Dp71 expression is associated with gastric adenocarcinoma prognosis. Oncotarget 2016;7:53702-53711.

17 Tan J, Tan S, Zheng H, Liu M, Chen G, Zhang H, Wang K, Tan S, Zhou J, Xiao XZ: HSF1 functions as a transcription regulator for Dp71 expression. Cell Stress Chaperones 2015;20:371-379.

18 Tan S, Tan S, Chen Z, Cheng K, Chen Z, Wang W, Wen Q, Zhang W: Knocking down Dp71 expression in A549 cells reduces its malignancy in vivo and in vitro. Cancer Invest 2016;34:16-25.

$>19$ Brambilla E, Negoescu A, Gazzeri S, Lantuejoul S, Moro D, Brambilla C, Coll JL: Apoptosis-related factors p53, Bcl2, and Bax in neuroendocrine lung tumors. Am J Pathol 1996;149:1941-1952. 


\section{Cellular Physiology Cell Physiol Biochem 2017;41:2523-2533

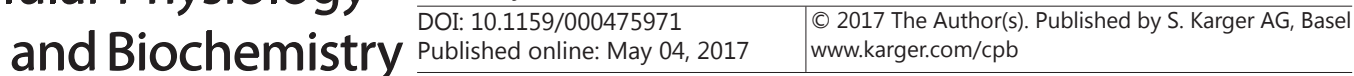 \\ Cheng et al.: Exploration of ZNF667 in HCC}

20 Martinez-Arribas F, Nunez-Villar MJ, Lucas AR, Sanchez J, Tejerina A, Schneider J: Immunofluorometric study of Bcl-2 and Bax expression in clinical fresh tumor samples from breast cancer patients. Anticancer Res 2003;23:565-568.

-21 Huang W, Li N, Hu J, Wang L: Inhibitory effect of RNA-mediated knockdown of zinc finger protein 91 pseudogene on pancreatic cancer cell growth and invasion. Oncol Lett 2016;12:1343-1348.

$\checkmark 22$ Song S, Christova T, Perusini S, Alizadeh S, Bao RY, Miller BW, Hurren R, Jitkova Y, Gronda M, Isaac M, Joseph B, Subramaniam R, Aman A, Chau A, Hogge DE, Weir SJ, Kasper J, Schimmer AD, Al-awar R, Wrana JL, Attisano L: Wnt inhibitor screen reveals iron dependence of beta-catenin signaling in cancers. Cancer Res 2011;71:7628-7639.

23 Yang L, Hamilton SR, Sood A, Kuwai T, Ellis L, Sanguino A, Lopez-Berestein G, Boyd DD: The previously undescribed ZKSCAN3 (ZNF306) is a novel "driver" of colorectal cancer progression. Cancer Res 2008;68:4321-4330.

-24 Yang L, Zhang L, Wu Q Boyd DD: Unbiased screening for transcriptional targets of ZKSCAN3 identifies integrin beta 4 and vascular endothelial growth factor as downstream targets. J Biol Chem 2008;283:35295-35304.

25 Zhang X, Jing Y, Qin Y, Hunsucker S, Meng H, Sui J, Jiang Y, Gao L, An G, Yang N, Orlowski RZ, Yang L: The zinc finger transcription factor ZKSCAN3 promotes prostate cancer cell migration. Int J Biochem Cell Biol 2012;44:1166-1173.

26 Chauhan S, Goodwin JG, Chauhan S, Manyam G, Wang J, Kamat AM, Boyd DD: ZKSCAN3 is a master transcriptional repressor of autophagy. Mol Cell 2013;50:16-28.

27 Bargou RC, Daniel PT, Mapara MY, Bommert K, Wagener C, Kallinich B, Royer HD, Dorken B: Expression of the bcl-2 gene family in normal and malignant breast tissue: low bax-alpha expression in tumor cells correlates with resistance towards apoptosis. Int J Cancer 1995;60:854-859.

28 Sakakura C, Sweeney EA, Shirahama T, Igarashi Y, Hakomori S, Tsujimoto H, Imanishi T, Ogaki M, Ohyama T, Yamazaki J, Hagiwara A, Yamaguchi T, Sawai K, Takahashi T: Overexpression of bax sensitizes breast cancer MCF-7 cells to cisplatin and etoposide. Surg Today 1997;27:676-679.

29 Wu C, Geng X, Wan S, Hou H, Yu F, Jia B, Wang L: Cecropin-P17, an analog of Cecropin B, inhibits human hepatocellular carcinoma cell HepG-2 proliferation via regulation of ROS, Caspase, Bax, and Bcl-2. J Pept Sci 2015;21:661-668.

30 Garcia EJ, Lawson D, Cotsonis G, Cohen C: Hepatocellular carcinoma and markers of apoptosis (bcl-2, bax, bcl-x): prognostic significance. Appl Immunohistochem Mol Morphol 2002;10:210-217.

-31 Guo LL, Xiao S, Guo Y: Detection of bcl-2 and bax expression and bcl-2/JH fusion gene in intrahepatic cholangiocarcinoma. World J Gastroenterol 2004;10:3251-3254.

32 Jheon AH, Ganss B, Cheifetz S, Sodek J: Characterization of a novel KRAB/C2H2 zinc finger transcription factor involved in bone development. J Biol Chem 2001;276:18282-18289.

33 Gou D, Wang J, Gao L, Sun Y, Peng X, Huang J, Li W: Identification and functional analysis of a novel human KRAB/C2H2 zinc finger gene ZNF300. Biochim Biophys Acta 2004;1676:203-209.

34 Razin SV, Borunova VV, Maksimenko OG, Kantidze OL: Cys2His2 zinc finger protein family: classification, functions, and major members. Biochemistry (Mosc) 2012;77:217-226.

-35 Burdach J, O'Connell MR, Mackay JP, Crossley M: Two-timing zinc finger transcription factors liaising with RNA. Trends Biochem Sci 2012;37:199-205.

-36 Tian C, Xing G, Xie P, Lu K, Nie J, Wang J, Li L, Gao M, Zhang L, He F: KRAB-type zinc-finger protein Apak specifically regulates p53-dependent apoptosis. Nat Cell Biol 2009;11:580-591.

-37 Tonnessen-Murray CA, Lozano G, Jackson JG: The Regulation of Cellular Functions by the p53 Protein: Cellular Senescence. Cold Spring Harb Perspect Med 2017;7:

38 Moreno CS, Matyunina L, Dickerson EB, Schubert N, Bowen NJ, Logani S, Benigno BB, McDonald JF: Evidence that p53-mediated cell-cycle-arrest inhibits chemotherapeutic treatment of ovarian carcinomas. PLoS One 2007;2:e441.

-39 Tang X, Huang J, Xiong H, Zhang K, Chen C, Wei X, Xu X, Xie Q, Huang R: Anti-Tumor Effects of the Polysaccharide Isolated from Tarphochlamys Affinis in H22 Tumor-Bearing Mice. Cell Physiol Biochem 2016;39:1040-1050.

40 Yang H, Xiong J, Luo W, Yang J, Xi T: 8-Methoxypsoralen Induces Intrinsic Apoptosis in HepG2 Cells: Involvement of Reactive Oxygen Species Generation and ERK1/2 Pathway Inhibition. Cell Physiol Biochem 2015;37:361-374. 